\title{
Viscoelastic Silk Fibroin Hydrogels with Tunable Strength
}

Danyu Yao, ${ }^{\mathrm{a}^{*}}$ Meiqi Li, ${ }^{\text {a }}$ Ting Wang, ${ }^{\mathrm{a}}$ Fangfang Sun, ${ }^{\mathrm{a}}$ Chang Su, ${ }^{\mathrm{b}}$ Tingchun Shi ${ }^{\mathrm{a}^{*}}$

a School of Automation, Hangzhou Dianzi University, Hangzhou 310018, Zhejiang, People's Republic of China.

b The Children's Hospital of Medical College, Zhejiang University, Hangzhou 310052, Zhejiang, People's Republic of China.

*Corresponding author: Danyu Yao

E-mail: yaodanyu@hdu.edu.cn

Address: School of Automation, Hangzhou Dianzi University, Hangzhou 310018, Zhejiang, People's Republic of China.

*Corresponding author: Tingchun Shi

E-mail: stc@hdu.edu.cn

Address: School of Automation, Hangzhou Dianzi University, Hangzhou 310018, Zhejiang, People's Republic of China. 
Figure S1: Deconvolution figures of lyophilized silk fibroin solution and hydrogels. A. NSF solution, B. SNL $\beta$ solution, C. SNL $\beta$ induced hydrogel, D. Ultrasound induced hydrogel. $\mathrm{R}^{2}>0.99$.
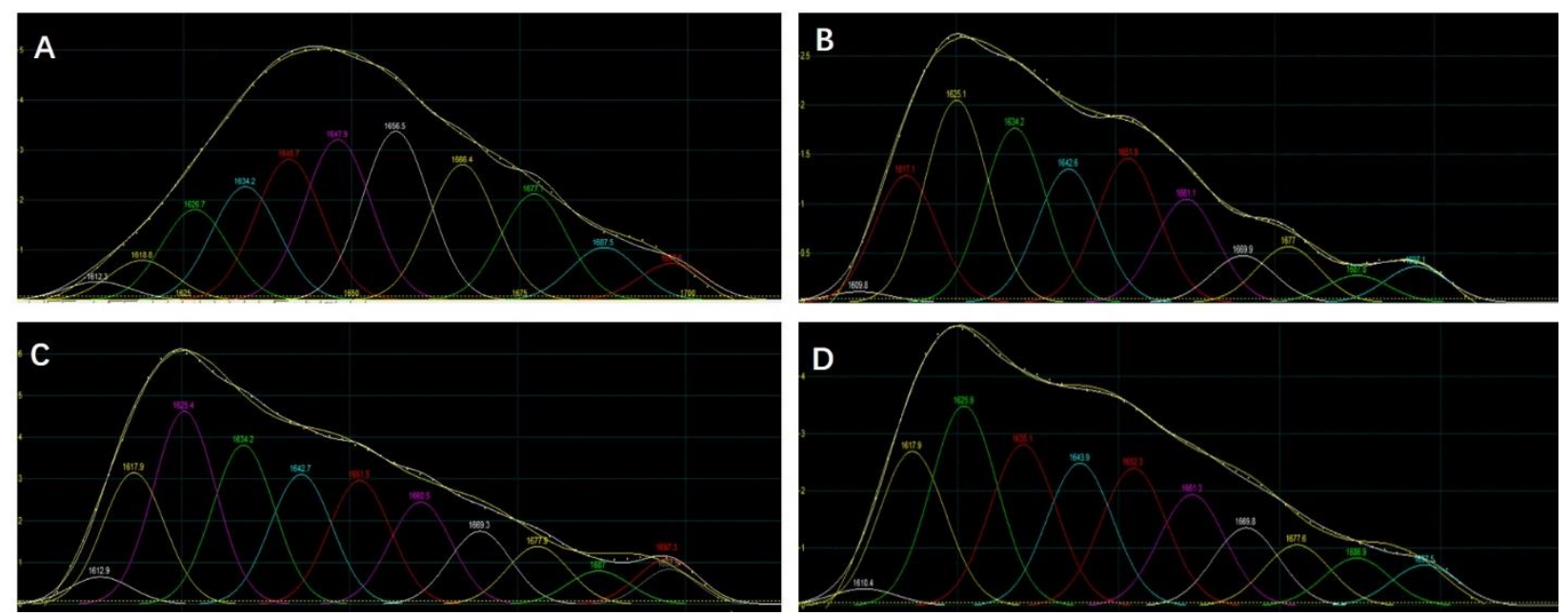
Figure S2: NSF solution (left) and SNL $\beta$ solution (right) stored at $4^{\circ} \mathrm{C}$ for 1 week or 4 weeks. Both NSF solution and SNL $\beta$ solution were stable and could be saved for more than 4 weeks without gelation.

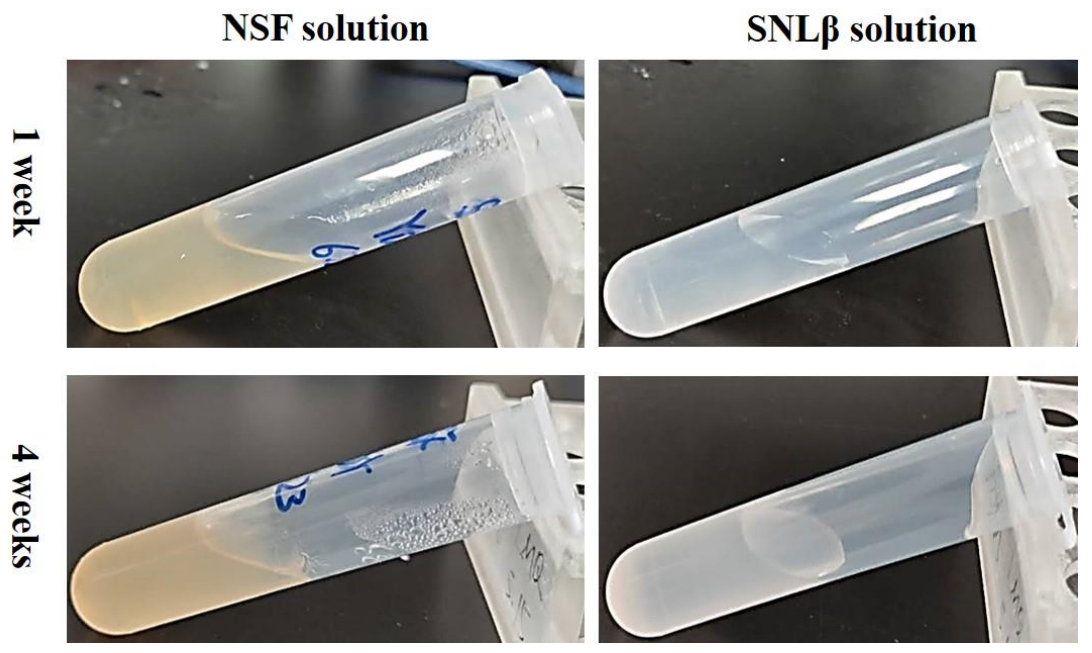


Figure S3: Mass remaining of silk fibroin hydrogels in PBS solution (A) and protease XIV solution (B). The hydrogels with a concentration of $1 \%$ and $2 \%$ were almost complete degraded in the enzyme solution within $72 \mathrm{~h}$. And the degradation rate decreased with the increase of the silk fibroin content both in PBS and the protease XIV solution. SNL $\beta / N S F=1: 9, n=4$.
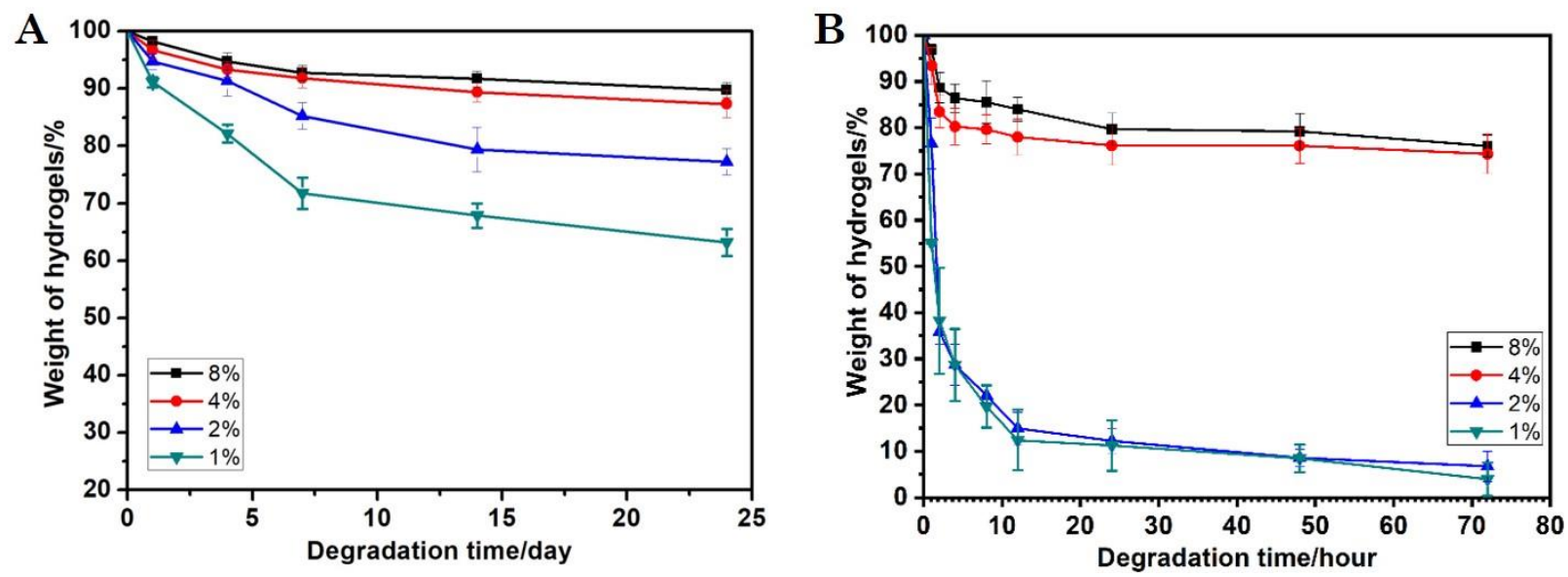
Figure S4: Morphology of the silk fibroin hydrogels before $(A, C)$ and after $(B, D)$ the compression. The traditional sonication-induced hydrogel (A, B) was crushed after the compression, while the morphology of SNL $\beta$ induced hydrogels $(\mathrm{SNL} \beta / \mathrm{NSF}=1: 9)$ still intact without cracks.

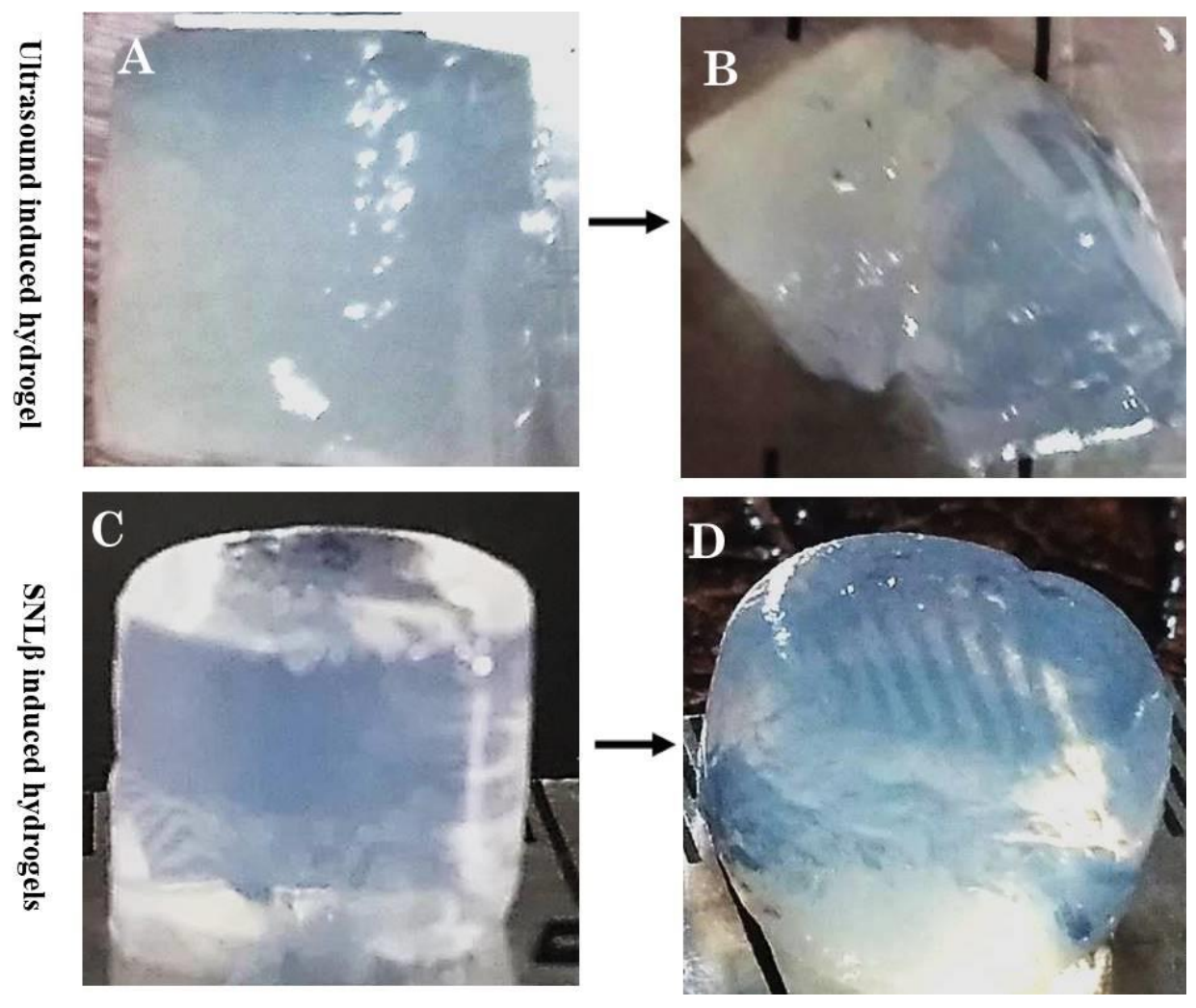


Figure S5 Gelatin was dissolved in water at $60 \square$ and remained in solution state at room temperature (left). While, once mixed with SNL $\beta$ solution (SNL $\beta /$ gelatin=1:4), the mixture converted into solid hydrogel within one minute although both gelatin solution and SNL $\beta$ solution were fluent solution (right).
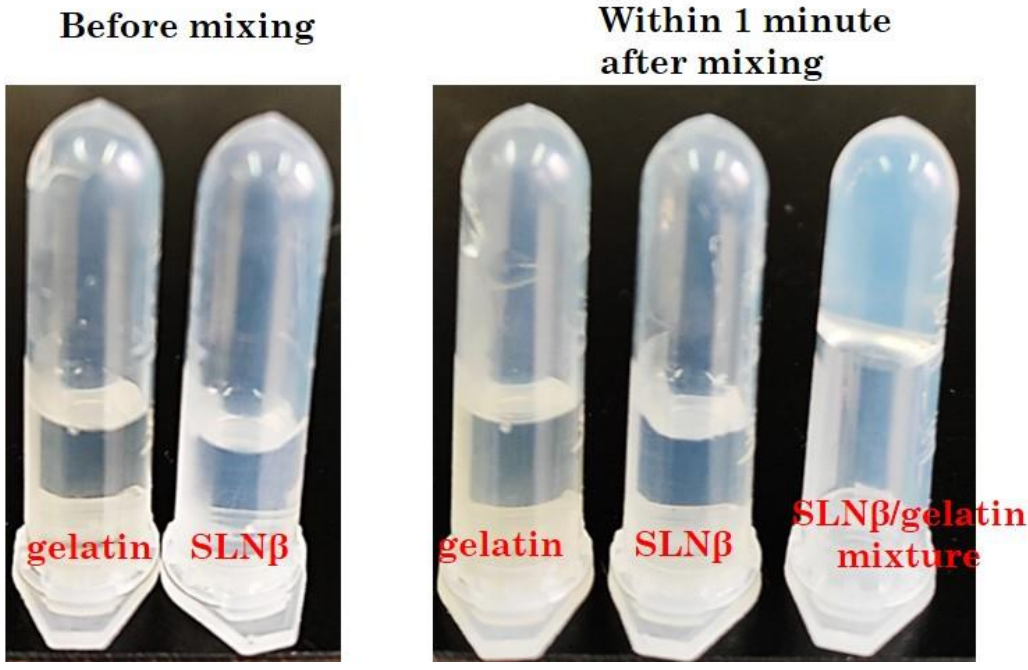